\title{
Benutzung 2018
}

Das Nutzungsverhalten in Bibliotheken unterliegt einem Wandel. Früher kam man in die Bibliothek, um sich reale Bücher nach Hause auszuleihen oder in die Lesesäle zu bestellen, Fernleihbestellungen aufzugeben, den Brockhaus im Lesesaal zu konsultieren, Zeitschriften zu lesen oder die Bibliothekarin persönlich um Rat zu fragen. Diese klassischen Benutzungssituationen gibt es heute zwar auch noch, aber der Trend geht ganz stark zur orts- und beratungsunabhängigen Literatur- und Informationsversorgung. Man nutzt das stets größer werdende Angebot von e-Ressourcen (e-journals, e-books, Datenbanken, Digitalisate) bequem von zu Hause, der Uni oder dem Arbeitsplatz aus. Das schlägt sich in der Statistik nieder. Über die Qualität der Rechercheergebnisse kann an dieser Stelle nur spekuliert werden.
Sammlungen führen. Im Vergleich zum Vorjahr ist ein Plus von $16 \%$ zu verzeichnen. Es liegt auf der Hand: Mit der Sichtbarkeit der Zimelien weltweit wird die Forschungsaktivität angekurbelt und intensiviert, weshalb mehr Unterstützungsleistungen durch spezialisierte Bibliothekare nötig werden. Die Unterhaltsträger sollten diese durch Bibliotheksleistungen verursachte Dynamik in der Forschung nicht gering schätzen und bei der Budgetierung der Digitalisierungsprozesse berücksichtigen. Erlaubt sei an dieser Stelle auch der Hinweis, dass Digitalisierung nicht ohne Langzeitarchivierung zu denken ist.

Abschließend noch der jährliche Blick auf die Benutzerstruktur. Er ist für die WLB, die funktional die Aufgabe einer Universitätsbibliothek für die

\begin{tabular}{l|l|r|r|}
\cline { 2 - 3 } Zur Erläuterung: & & $\mathbf{2 0 1 7}$ & $\mathbf{2 0 1 8}$ \\
\cline { 2 - 3 } Im Jahr 2018 & Allgemeine Angaben zur Benutzung & & \\
hatten 37.175 & Aktive Benutzer & 26.532 & 24.266 \\
Personen einen & Gültige Bibliotheksausweise & 37.788 & 37.175 \\
gültigen Biblio- & Entleihungen & 1.475 .582 & 1.412 .122 \\
theksausweis. & Verlängerungen & 969.223 & 924.837 \\
Aktive Benutzer & Auskunftsanfragen Info & 21.275 & 19.546 \\
sind diejenigen, & Aktive Fernleihe & 76.038 & 70.537 \\
die mindestens & Passive Fernleihe & 18.538 & 17.166 \\
einmal im Jahr & Besucher Hauptlesesaal & 258.492 & 276.343 \\
ein Buch auslei- & Besucher Fachlesesäle & 10.862 & 9.889 \\
hen, das waren & Zugriffe & & \\
in 2018 24.266 & OPAC + Portal & 1.762 .872 & 1.765 .612 \\
Personen. Wel- & Datenbanken, e-journals, e-books (HAN) & 161.446 & 171.310 \\
che Services kon- & Digitale Sammlungen WLB & 602.791 & 812.730 \\
\cline { 2 - 3 } & &
\end{tabular}

Geistes- und Sozialwissenschaften in Stuttgart wahrnimmt, obligatorisch. Denn es gilt, ein bedarfsgerechtes Literatur- und Schulungsangebot anzubieten, ohne den langfristigen, nachhaltigen Bestandsaufbau zu vernachlässigen. Wie erwartet, gibt kret nachgefragt wurden, kann nicht beantwortet werden, aber die Vermutung liegt nahe, dass ein Großteil dieses Personenkreises v.a. die WLAN-Infrastruktur des Hauses bzw. elektronische Medien nutzten.

Festzustellen ist ebenfalls, dass das zunehmende Angebot in den Digitalen Sammlungen ${ }^{1}$ der WLB vermehrt zu Nachfragen bei den Historischen es nur marginale Verschiebungen innerhalb der Studierendengruppen, auch dieses Jahr, wie in den vergangenen Jahrzehnten liegt die Quote der Studierenden bei $52 \%$. Wenn alle Auszubildenden und Schüler miteinbezogen werden, liegt der Wert sogar bei $73 \%$. Das ist Grund und Bestätigung für die WLB, den Fokus auch auf die Nachwuchswissenschaftler zu richten.

Martina Lüll 